\title{
Vibration Control of Flexible Spacecraft Using Adaptive Controller.
}

\author{
V.I.George", B.Ganesh Kamath ${ }^{\#}$, I.Thirunavukkarasu\#, Ciji Pearl Kurian* \\ \#ICE Department, Manipal University, MIT.Manipal, 576104, India, \\ E-mail:vig_rect@yahoo.com,it_arasu@yahoo.co.in \\ ${ }^{*} E \& E$ Dept Manipal University, MIT Manipal,576104, India \\ E-mail:cpk001@yahoo.com
}

\begin{abstract}
The aim is to develop vibration control of flexible spacecraft by adaptive controller. A case study will be carried out which simulates planar motion of flexible spacecraft as a coupled hybrid dynamics of rigid body motion and the flexible arm vibration. The notch filter and adaptive vibration controller, which updates filter and controller parameters continuously from the sensor measurement, are implemented in the real time control. The least mean square algorithm using the adaptive notch filter is applied to the flexible spacecraft. This study will show that the adaptive vibration controller successfully stabilizes the uncertain and it will accurately control the vibration of flexible spacecraft. The Least mean square algorithm is applied in flexible spacecraft to attenuate the vibration. The simulation studies are carried out in a Matlab/Simulink environment.
\end{abstract}

Keywords - Adaptive control; Least mean square; Recursive least squares; Flexible spacecraft.

\section{INTRODUCTION}

Adaptive is the gradual process of adjusting to new physical conditions exhibited by living organisms. In control, it is any control scheme that changes its structure and/or parameters to maintain a consistent performance despite environmental changes [1-5].

Flexible components interact with the spacecraft attitude control system by superimposing deflection and acceleration on the angular measurement made by attitude sensor and rate gyros, the control system can give erroneous command signal that could destabilize a spacecraft.

Flexibility can also move the instantaneous centre of mass and moments of inertia induce unexpected responses to command control torques. The disturbances are external torques which affects overall system performance. They can be either deterministic or random in nature. The torque produced by the impact of meteoroids is randomly distributed. The paper is organized as Section II explains rigid body controller, Section III discuss vibration controller, Section IV gives the vibration control algorithm, Section V Least Mean Square (LMS) vibration controller using notch filter, Section VI three axis flexible spacecraft control, Section VII conclusion and references are in Section VIII.

\section{Rigid BODY CONTROLLER}

In this section, an attitude controller is using the adaptive control algorithm for the flexible spacecraft model is addressed. The PD/PID controller is designed as a baseline controller for flexible spacecraft attitude control. However, the performance of the PD/PID controller degrades when the external persistent disturbance exists. In the following section, the vibration controller is designed to consider the external disturbance. The adaptive controller shows a good performance with respect to disturbance. Figure(1) shows the overall architecture of the adaptive control system. The reaction wheel module actuated by a DC motor is modeled as a following second-order system. Controller input :

$$
\tau=\tau_{v}+\tau_{c}=\tau_{v}+k_{p} \boldsymbol{q}_{e}+\boldsymbol{k}_{d} \dot{q}_{e}
$$

where the $\boldsymbol{\tau}_{\boldsymbol{c}}$ is the PD/PID controller output of the rigid body, the $\boldsymbol{\tau}_{v}$ is the vibration controller output.

\section{VIBRATION CONTROLLER}

Adaptive controller is designed to control the flexible spacecraft with persistence disturbance. For the vibration control of spacecraft, the least mean square (LMS) algorithm using notch filter is applied to the flexible spacecraft. 


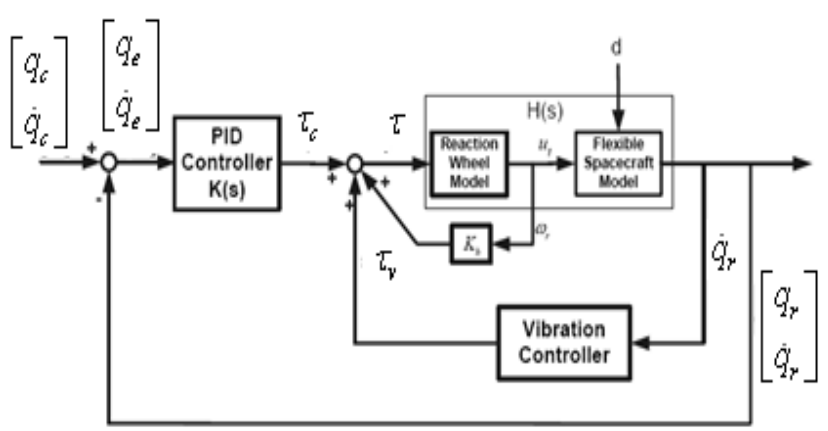

Fig.1: Attitude Controller structure

The feed forward LMS algorithm generally needs a reference signal. The notch filter estimates the external disturbance signal frequency and the reference signal is generated by this estimated frequency. A block diagram of the adaptive vibration controller which will be considered in this section is shown in Figure(2).

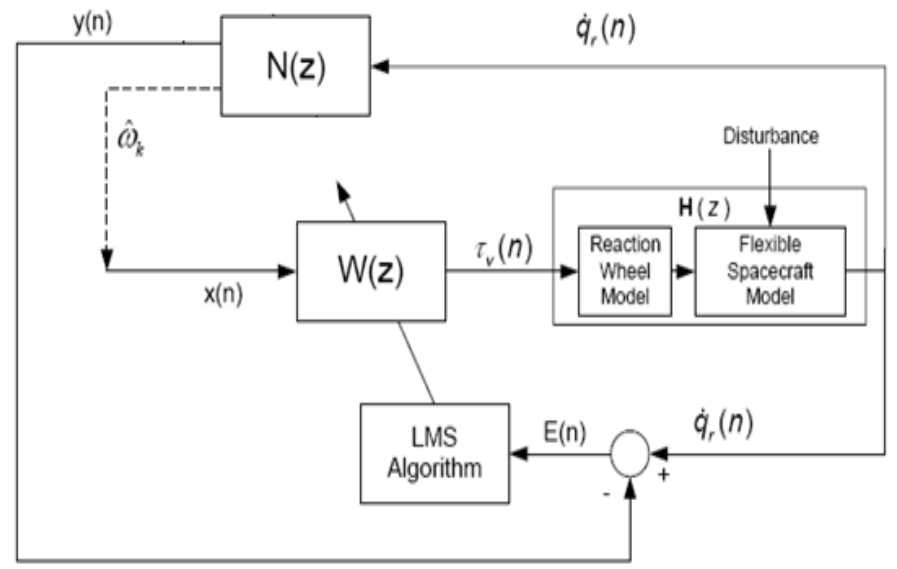

Fig.2: Block diagram of active vibration controller.

\section{VibRATION CONTROL AlgORITHM}

The RLS Filter[6] block recursively computes the least squares estimate (RLS) of the FIR filter weights and acts as notch filter in this case. The block estimates the filter weights or coefficients, needed to convert the input signal into the desired signal. Connect the signal which wants to filter to the Input port. Connect the signal which want to model to the desired port.The desired signal must have the same data type, frame status, complexity, and dimensions as the input signal. The Output port outputs the filtered input signal, which can be sample or frame based. The Error port outputs the result of subtracting the output signal from the desired signal. The figure 3 explains the SIMULINK block diagram of vibration control algorithm. This filtered input signal is taken as reference signal to the LMS filter and then disturbance signal will remain same as that of RLS filter input. The output signal will be better than input signal. The filtered input signal and output of the LMS filter is shown in figure (4) and figure (5).

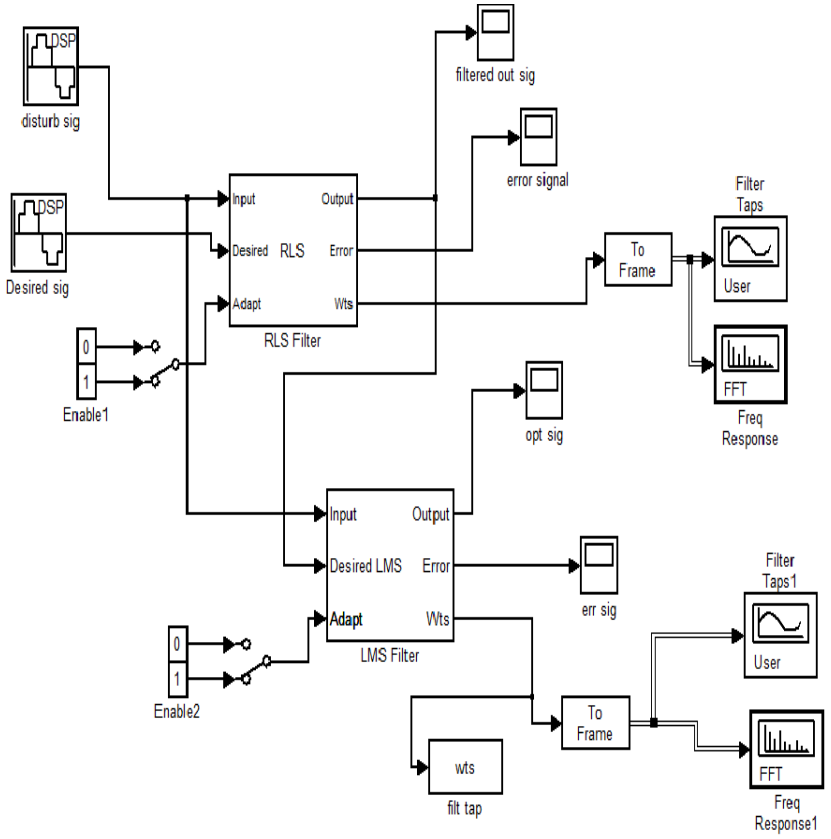

Fig 3: Vibration Control Algorithm

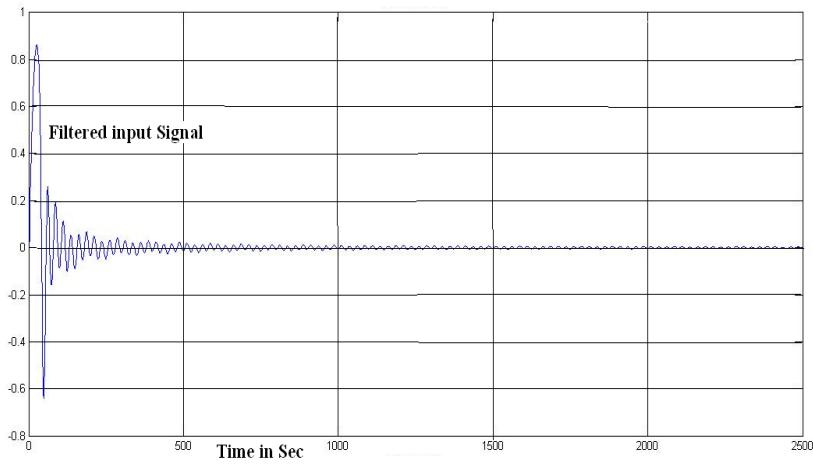

Fig 4: Filtered input signal

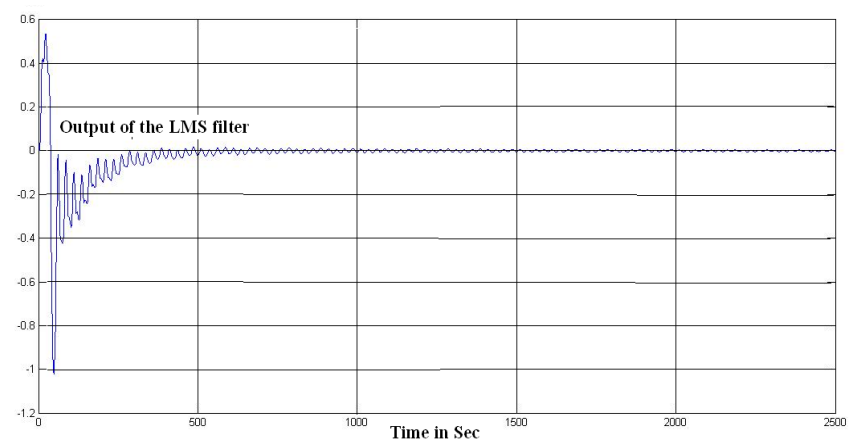

Fig 5: output of the LMS filter

Rate control using vibration controller.

By introducing the vibration controller algorithm the angle rate of the spacecraft is controlled. But the rigid body controller is controlled by PD attitude controller whereas flexible body is controlled by the vibration controller. Attitude control using vibration control is shown in figure (6). Vibration control of rate signal is shown in figure (7). 


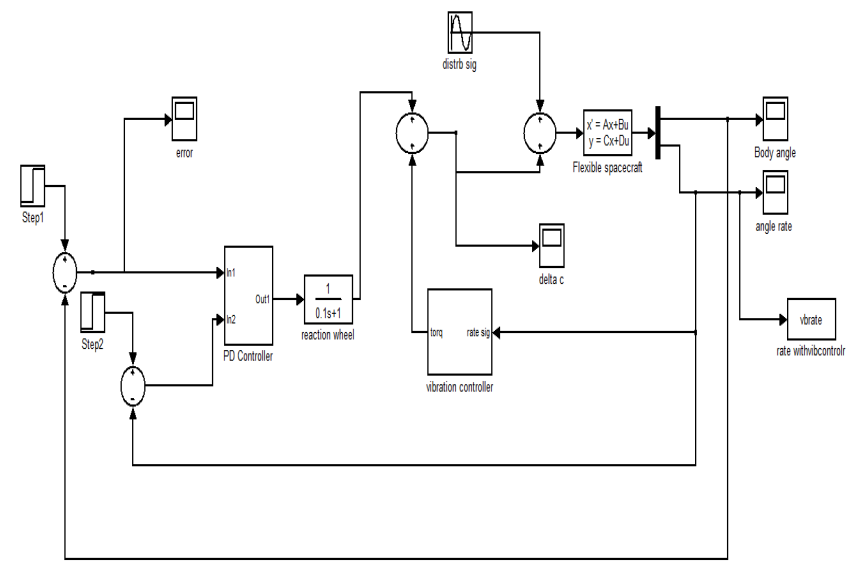

Fig 6: Attitude control using Vibration control

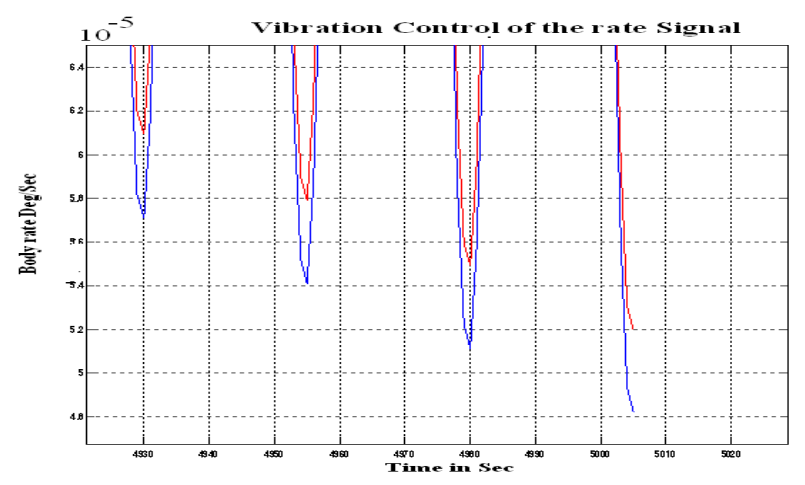

Fig.7: Comparison of rate signal with and without controller

\section{LMS VIBRATION CONTROLLER USING NOTCH FILTER}

The notch block $N(z)$ in Fig.2. is explained in this section. The multi-mode notch filter in the ARMA structure is required for matrix calculation. Hence, it is difficult to apply it to a real time system for multiple sinusoidal signals. To reduce the computational time, a cascade of the second-order notch filters is designed as shown in Figure.8. When the number of the disturbance signal to be rejected is equal to $p$, each notch frequency is determined by the corresponding second-order notch filter in the cascade.

The disturbance signal generated by a vibration generator on the tip of the spacecraft flexible beam. The disturbance frequencies equal to the spacecraft first and second mode.

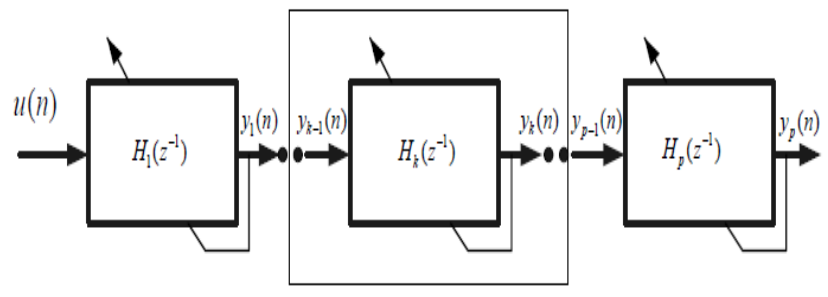

Fig 8: Cascade of second-order notch sections

The attitude control of flexible spacecraft is obtained by the vibration control. The least mean square algorithm is applied to many systems to attenuate the vibration. In this study the vibration controller is the LMS using the notch filter algorithm. The spacecraft mode frequencies are estimated using RLS algorithm in the modified cascade type notch filter.
In figure $2 \dot{q}_{r}(n)$ is the input signal of the notch filter, The $\dot{q}_{r}(n)$ is the rate signal of the flexible spacecraft and estimated frequency is updated using the rate sensor signal. The rate sensor measures the rigid body signal as well as disturbance. Control system developed for rigid spacecraft can become very well closed loop unstable, when flexible appendages come in to picture. It is necessary to satisfy the stability condition for flexible spacecraft. Only the rate of attitude motion and actuator providing attitude torque are considered. Adaptive filter is used to remove the noise from an unknown system. The figure (9) Illustrates the adaptive noise cancellation diagram.

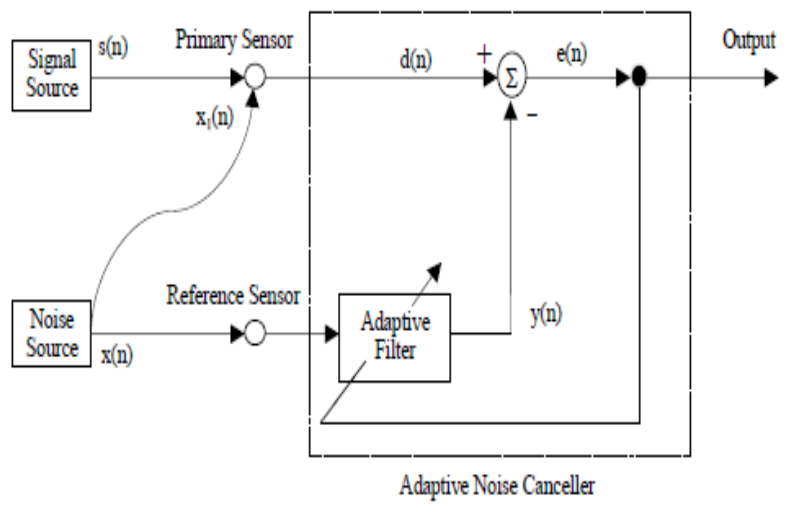

Fig. 9: Adaptive noise cancellation diagram.

So long as the input noise to the filter remains correlated to the unwanted noise accompanying the desired signal, the adaptive filter adjusts its coefficients to reduce the value of the difference between $y(k)$ and $d(k)$, removing the noise and resulting in a clean signal in $e(k)$. Notice that in this application, the error signal actually converges to the input data signal, rather than converging to zero.

\section{Design of Notch Filters}

Here three sine waves of different frequencies are used .The Notch filter notches out some of the frequency and separates the desired frequency. Adaptive technique is not implemented but by considering three bending modes of the spacecraft are removed.

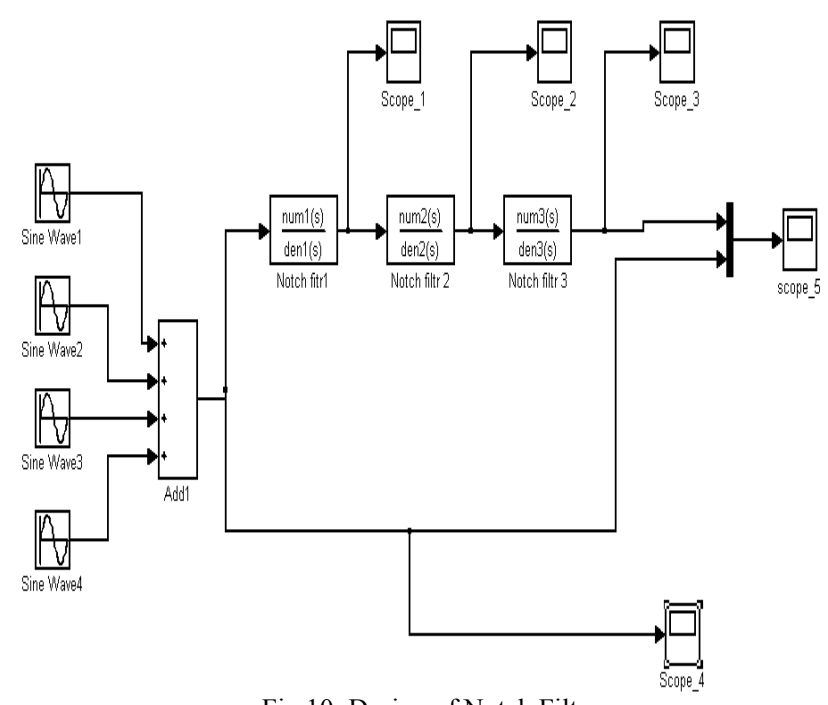

Fig 10: Design of Notch Filter 

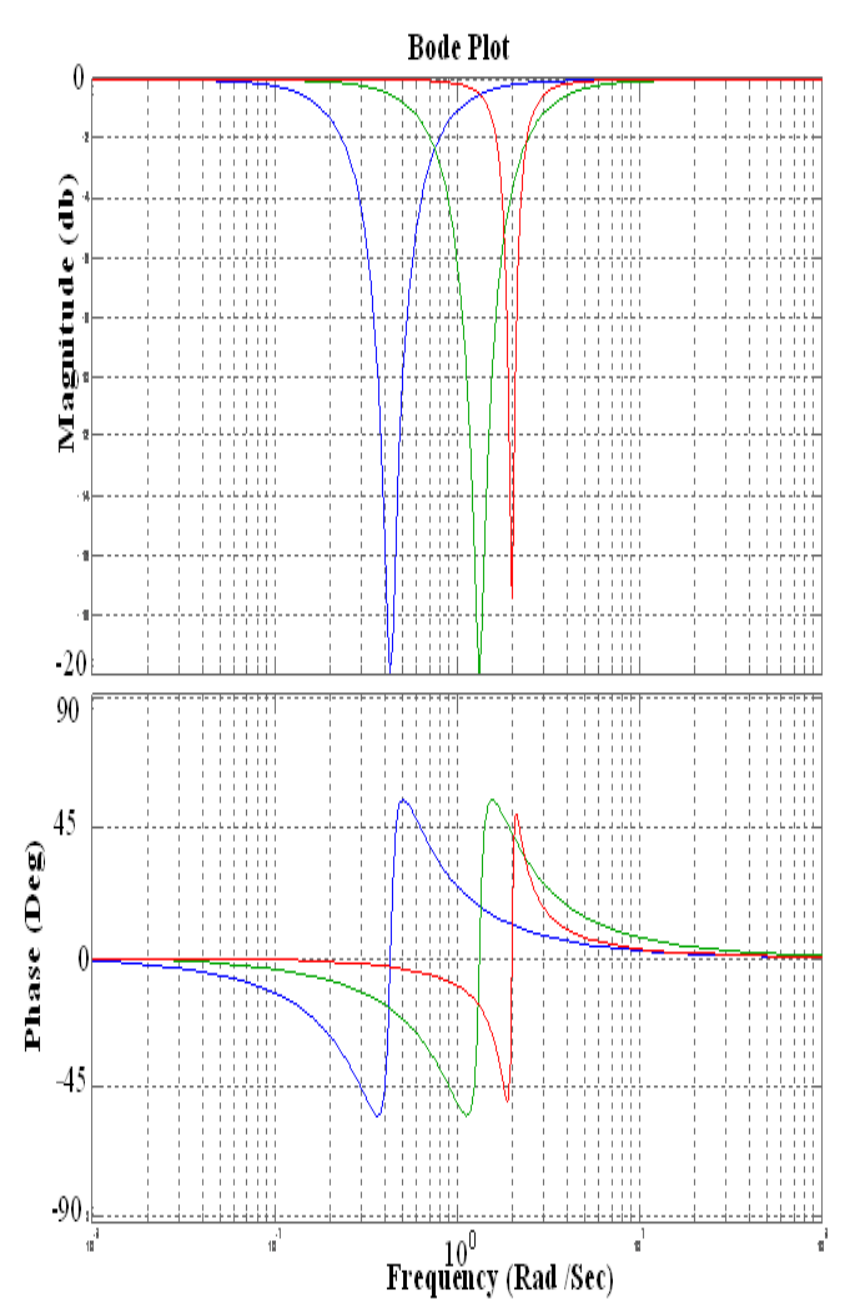

Fig 11: Bode plot of Notch Filter

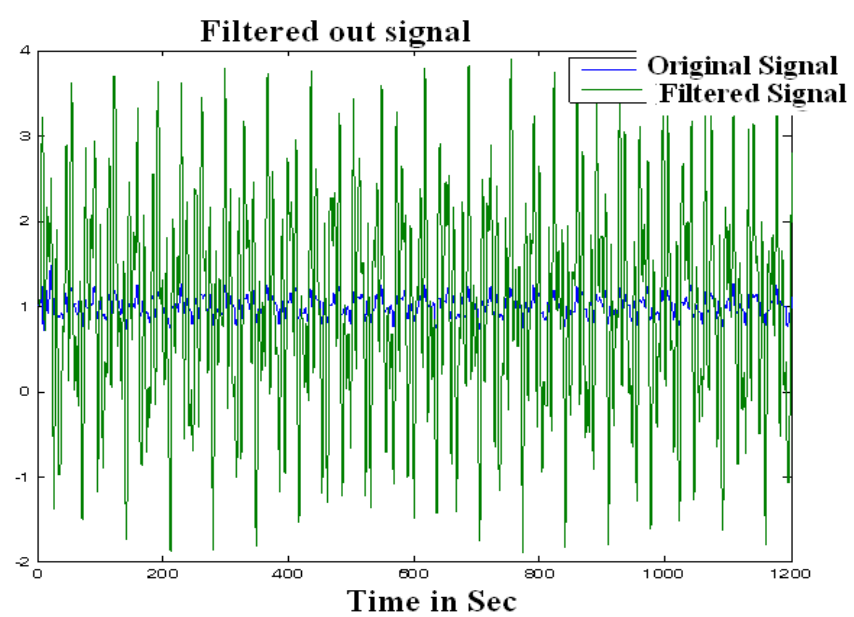

Fig.12: Filtered out signal of the Notch filter in general

This explains about the notch sections introduced in place of RLS filter and that filtered signal is fed into the desired signal of the LMS filter which consists of pure disturbance signal. When this signal is fed into the original rate signal of the spacecraft. The much improved version of rate signal is obtained. The figure (10) gives the design of notch filter figure (11) Bode phase magnitude plot of notch filter and figure (12) gives the filtered output signal of the notch filter. Filtered output of the notch filter is shown in figure (13). Using notch section the disturbance signal is estimated as shown in figure (14).

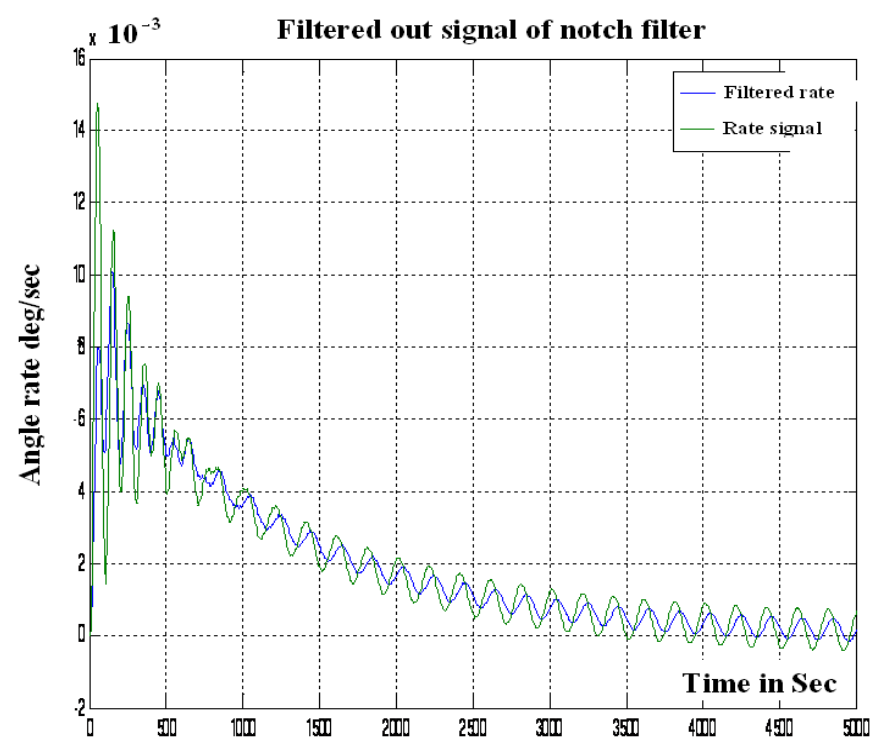

Fig.13: Notch Filter output

\section{THREE AXIS FlEXIBLE SPACECRAFT CONTROLS}

The design is extended for three axis flexible spacecraft wherein the final value of the attitude is 1 and final value of the rate is 0 . The SIMULINK diagram of a 3 axis flexible body spacecraft is shown in figure (15) Here ideal control of the spacecraft is considered .The actuator dynamics are not involved instead the control signal is directly fed into the Space craft.

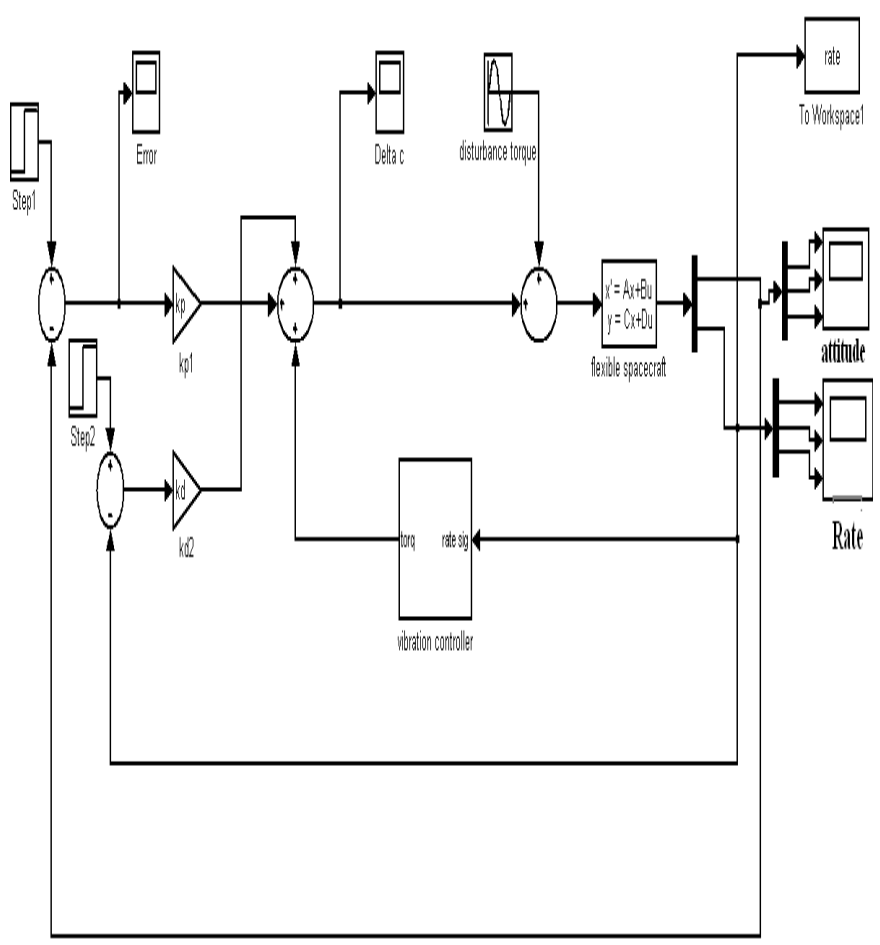

Fig 15: Response of three axis flexible body 


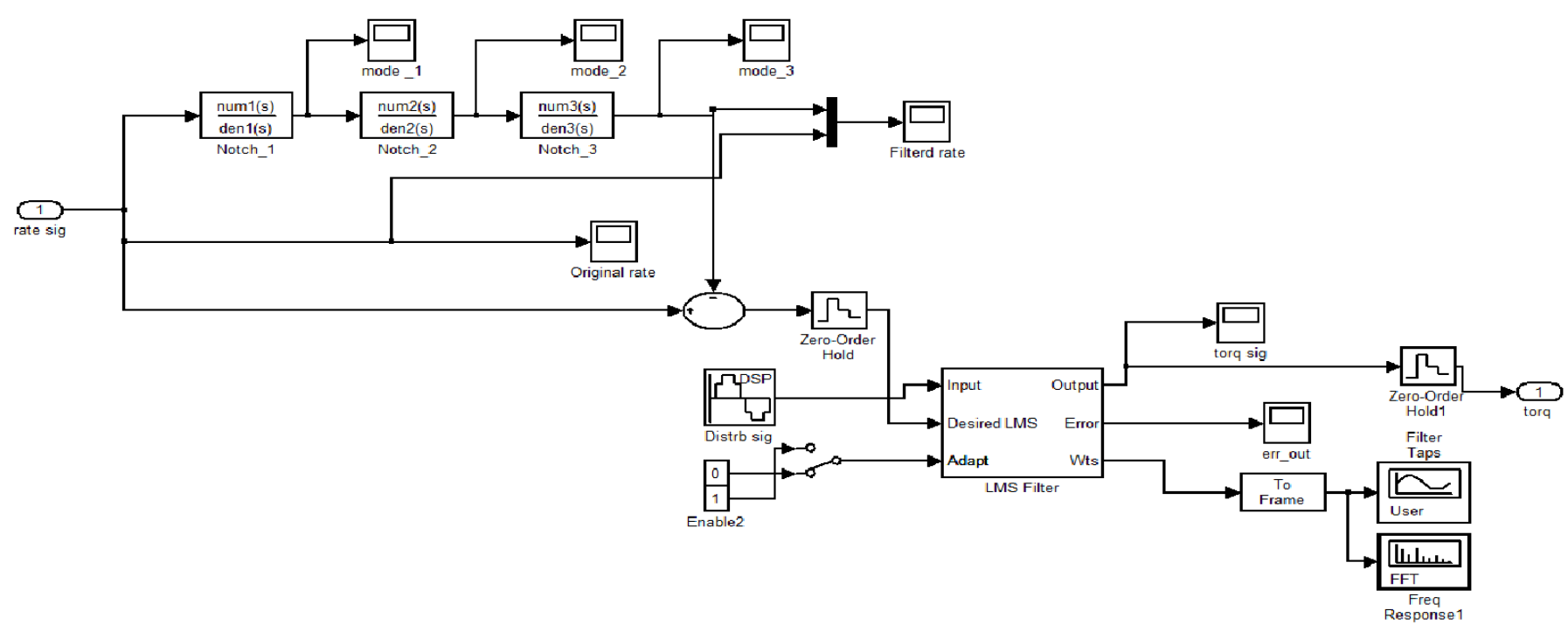

Fig14: Using notch section the disturbance signal is estimated

Table 1 gives the parameters of the 3 axis spacecraft and controller. The simulation results shown in figure (15) the yaw, roll, pitch rate converges to zero. Figure (16) yaw, roll, pitch attitude of the space craft converges to 1 .
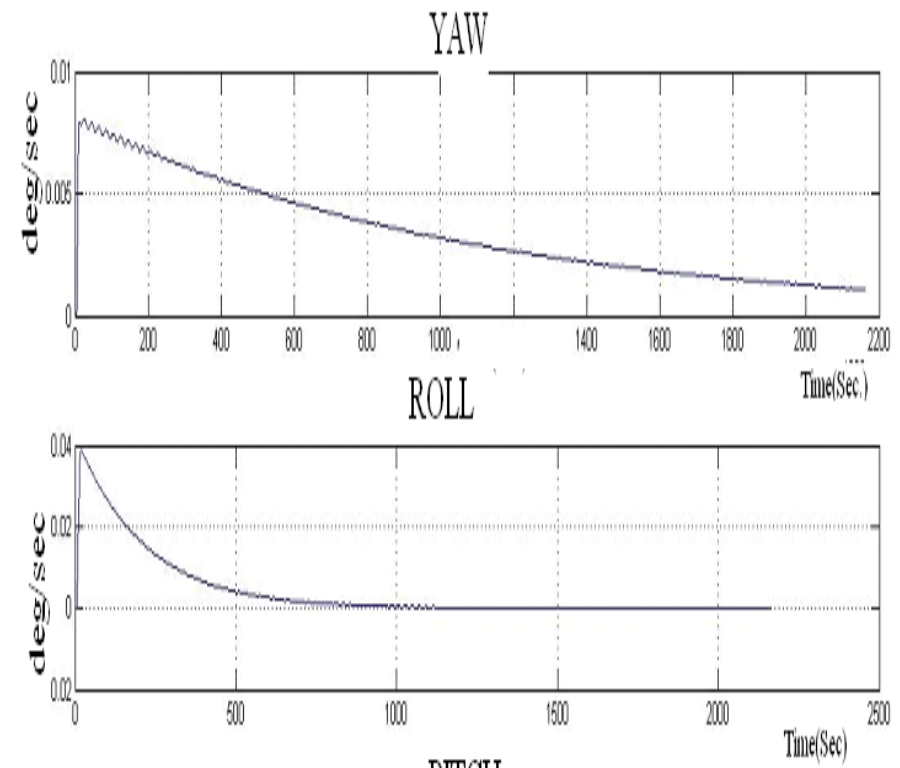

PITCH

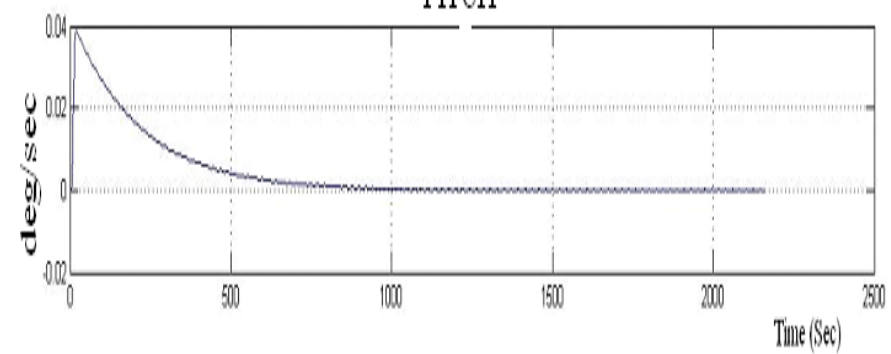

Fig 16: Rate of the spacecraft converges to zero
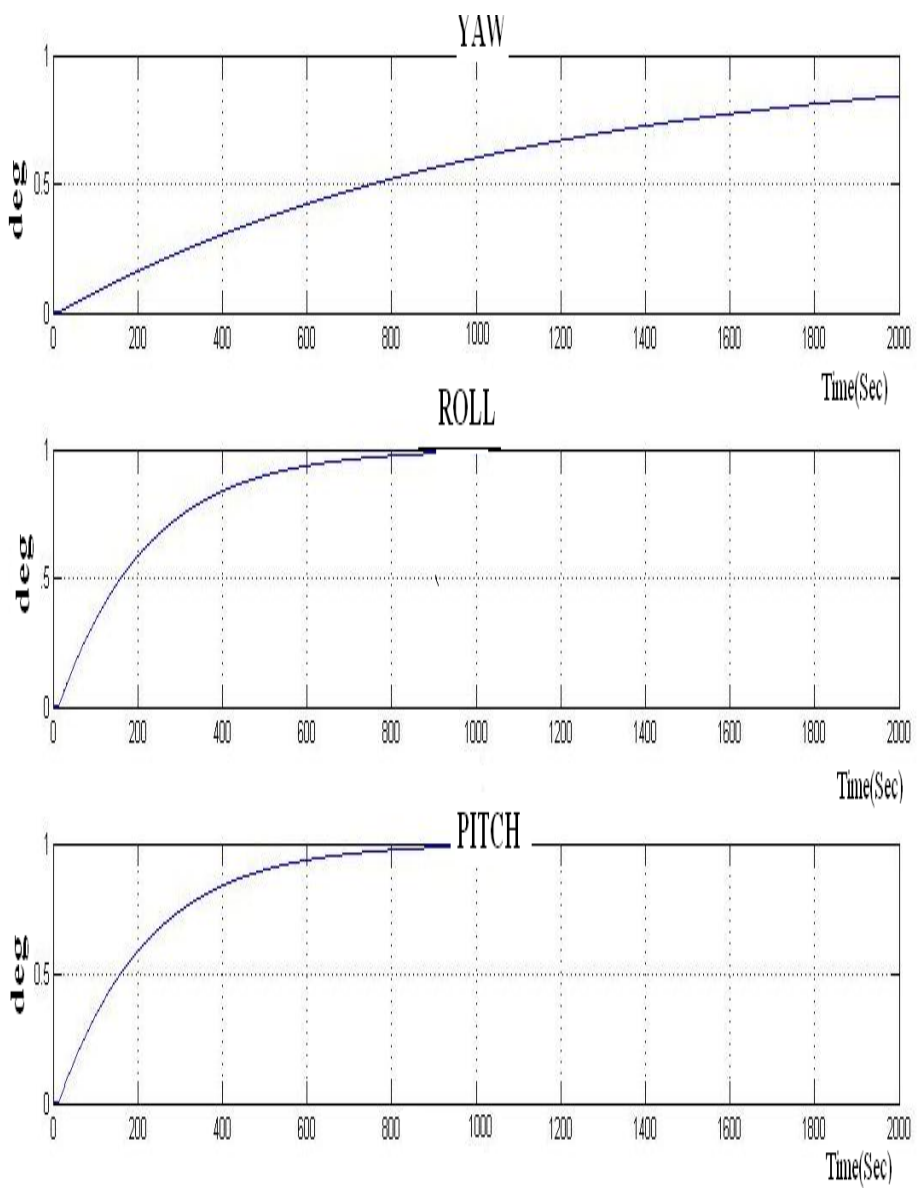

Fig 17: Attitude of the spacecraft converges to one. 
TABLE 1

PARAMETERS OF THE 3 AXIS SPACECRAFT AND CONTROLLER.

\begin{tabular}{|c|c|}
\hline Parameter & Values \\
\hline Inertia(kgm^2 2$)[\mathrm{Jx} \mathrm{Jy} \mathrm{Jz}]^{\prime}$ & {$[1920.6,1419.1,1025.6]$} \\
\hline$\xi$ & 0.002 \\
\hline ts & $100 \mathrm{~s}$ \\
\hline
\end{tabular}

\section{CONCLUSIONS}

The objective is the vibration control of flexible space craft using adaptive controller and its significance were met. The RLS and LMS algorithms are developed and tested. The vibration controller using LMS algorithm has been designed successfully to attenuate the disturbance signal. This is main advantage of the vibration control in eliminating sinusoidal disturbance.

The PD attitude control of the spacecraft is done successfully by considering disturbance signal as first bending mode frequency and number of flexible mode as one. Here on-line estimation of the disturbance signal is not carried out rather the disturbance signal is fed directly into the LMS as a reference signal. The same is carried out by designing a cascade notch filter by taking first mode alone as well as first three flexible modes also. The vibration control of flexible spacecraft was found to be 35 to 40 percent reduction in steady state of the system outputs especially in rate output. The cascaded notch filter was successfully used to eliminate the multiple frequencies of the flexible spacecraft. The vibration control using LMS with RLS filter as notch filter can accurately control the attitude of the flexible spacecraft at steady state. The simulation results illustrate the feasibility of the vibration controller for the flexible spacecraft stabilization and flexible effect on spacecraft attitude dynamics. The above mentioned design is carried out for three axes attitude stabilization case with ideal case also and the results are found to be satisfactory.

\section{NOMENCLATURE}

$\boldsymbol{\tau}_{\boldsymbol{c}} \quad$ PD/PID controller output of the rigid body

$\boldsymbol{\tau}_{v} \quad$ Vibration controller output.

d Disturbance

$\dot{q}_{r}(n)$ Input signal of the notch filter (rate signal)

$\mathrm{N}(\mathrm{z}) \quad$ Notch block

$\xi \quad$ Damping ratio

$\mathbf{t}_{\mathbf{s}} \quad$ Settling time

$\mathrm{J}_{\mathrm{X}}, \mathrm{J}_{\mathrm{Y}}, \mathrm{J}_{\mathrm{Z}}$ Moment of Inertias.

RLS Recursive least square

LMS Least mean square

\section{REFERENCES}

[1] Preumont. A Vibration Control of Active Structures, 2nd ed., Kluwer Academic Publishers, 2002.

[2] Lim,T.W, Bosse A, Fisher, S . Adaptive Filters for Real-Time, System Identification and Control AIAA Journal of Guidance, Control, and Dynamics, Vol. 20, January 1997 pp. 61-66.

[3] Joshy S.M , Control of Large Flexible Space Structures, Springer Verlag, 1989.

[4] Albassam, B. A Optimal Near-Minimum-Time Control Design for flexible structures , AIAA Journal of Guidance,Control, and Dynamics, Vol. 25, July 2002, pp. 618-625

[5] Choi , H.D., and Bang H.C, An Adaptive Control Approach to the Attitude Control of a Flexible Rocket, Control Engineering Practice, August, 2000, pp.1003-1010.

[6] M. Wegmuller, J. P. von der Weid, P. Oberson, and N. Gisin, "High resolution fiber distributed measurements with coherent OFDR," in Proc. ECOC'00, 2000, paper 11.3.4, p. 109.

[7] T.Ramano , J.M Bellanger .M, Fast Least Square Adaptive Notch Filtering" ,IEEE transaction on Acoustic speech signal processing, Vol 36, Sept 1988, pp, 1536-1540.

[8] Rao. DV, Kung S.Y, Adaptive Notch Filtering for the retrieval of sinusoids in Noise", IEEE transaction on Acoustic speech signal processing, Vol 32,Aug 1984, pp791-802.

[9] Haykin S, Adaptive filter theory $4^{\text {th }}$ ed. Prentice Hall NJ, Chapter 9, 2002 . 